\title{
Uma agenda de pesquisa para as Ciências Sociais e Humanas em tempos de pandemia da COVID-19
}

\author{
A research agenda for the Social and Human \\ Sciences during the COVID-19 pandemic
}

\author{
Una agenda de investigación para las Ciencias \\ Sociales y Humanas durante la pandemia \\ COVID-19
}

\author{
Mônica de Oliveira Nunes 1 \\ Suely Deslandes 2 \\ Marta Inez Machado Verdi 3 \\ Rui Massato Harayama 3,4 \\ Luís Henrique da Costa Leão 5 \\ Martinho Braga Batista e Silva 6 \\ Tatiana Engel Gerhardt 7
}

doi: 10.1590/0102-311X00158421

O ano de 2020 marcou o crescimento global da doença provocada pelo SARS-CoV-2, a COVID-19, acompanhada pela intensificação de publicações científicas 1. O ano encerrou com mais de 250 mil artigos produzidos mundialmente e, em outubro de 2020, o Brasil aparecia no 11 lugar do ranking de publicações 2. Paradoxalmente, o ano foi marcado por cortes expressivos no financiamento atribuído pelas agências de fomento à ciência brasileira, a despeito da necessidade de pesquisas para o enfrentamento dos diversos problemas causados pela pandemia. Esse direcionamento se evidenciou desde 9 de março de 2020, com a Portaria no 343 da Coordenação de Aperfeiçoamento de Pessoal de Nível Superior (CAPES) com diminuição do quantitativo de bolsas de pós-graduação, reduzindo-as em grau decrescente segundo a nota dos Programas de Pós-graduação (PPGs), atingindo fortemente os PPGs com notas menores, cuja atuação é estratégica para seus âmbitos regionais.

Nesse período, também o Conselho Nacional de Desenvolvimento Científico e Tecnológico (CNPq) anunciou corte orçamentário, afetando pesquisas aprovadas ou em curso, além de ameaçar com a não abertura do Edital Universal de 2020. Em reunião com a diretoria da Sociedade Brasileira para o Progresso da Ciência (SBPC), o presidente do CNPq apontou para o "mercado" como alternativa de recomposição de recursos financeiros para pesquisas, evidenciando que essa articulação estava associada ao desenvolvimento ou fortalecimento de "áreas estratégicas" 4 . O posicionamento indica que seriam estratégicas apenas as pesquisas que respondessem aos interesses do "mercado", este materializado nas empresas ou indústria, englobando a concessão de bolsas. É evidente que as Ciências Sociais e Humanas (CSH) não fazem parte desses "grupos prioritários", tendo em vista que muitas temáticas por elas privilegiadas - racismo, desigualdade social, relações de gênero, democracia, movimentos sociais e tantas outras - não coadunam com as necessidades tornadas estratégicas na lógica do mercado. A consequência é a ausência de edital específico para as CSH e, em eventuais editais lançados, a participação seria de forma subsidiária, investigando aspectos que convergissem com interesses do mercado.

Desenhada esta conjuntura adversa à produção da ciência e tecnologia brasileira de modo mais largo, e das CSH de modo particular, a sociedade científica, nas suas representações, intensificou movimentos para enfrentar esse desmonte e defender sua recomposição. Observaram-se mobilizações realizadas pela SBPC, pelas entidades de Saúde Coletiva e de Bioética, entre outras, além de
1 Instituto de Saúde Coletiva, Universidade Federal da Bahia, Salvador, Brasil.

2 Instituto Nacional de Saúde da Mulher, da Criança e do Adolescente Fernandes Figueira, Fundação Oswaldo Cruz, Rio de Janeiro, Brasil. 3 Universidade Federal de Santa Catarina, Florianópolis, Brasil.

4 Universidade Federal do Oeste do Pará, Santarém. Brasil.

5 Instituto de Saúde Coletiva, Universidade Federal de Mato Grosso, Cuiabá, Brasil. 6 Instituto de Medicina Social, Universidade do Estado do Rio de Janeiro, Rio de Janeiro, Brasil. 7 Universidade Federal do Rio Grande do Sul, Porto Alegre, Brasil.

\section{Correspondência}

M. O. Nunes Instituto de Saúde Coletiva, Universidade Federal da Bahia.

Rua Basílio da Gama s/n, Salvador, $B A$ 40110-040, Brasil. nunesm@ufba.br 
associações da área das Humanidades, como a Associação Nacional de Pós-graduação e Pesquisa em Ciências Sociais (ANPOCS) e a Associação Brasileira de Antropologia (ABA). A crescente mobilização reuniu mais de 60 entidades que assinaram uma carta, solicitando a revogação da Portaria no 34/2020 da CAPES, subscrita também pela Associação Brasileira de Saúde Coletiva (Abrasco).

Esse movimento redundou em êxito parcial. Sobre a destituição das CSH como áreas prioritárias, obtém-se uma primeira vitória com a flexibilização do Ministério da Ciência, Tecnologia, Inovações e Comunicações (MCTIC) que, através da Portaria no 1.329 5, de 27 de março de 2020, reconheceu a "característica essencial e transversal" da área de CSH. Esse reconhecimento, entretanto, até hoje não se traduziu em termos orçamentários. Já a Portaria no 34/2020 da CAPES, apesar da ação do Ministério Público Federal (MPF), que recomenda a sua revogação, ainda segue vigente.

Em 20 de abril de 2020, a Abrasco publicou a nota Precisamos das Ciências Sociais e Humanas para Compreender e Enfrentar a Pandemia da Covid-196. Imediatamente o Núcleo Gestor da Comissão de Ciências Sociais e Humanas em Saúde da Abrasco (CCSHS/Abrasco) convocou seus representantes de todos os PPGs em Saúde Coletiva para discutir a situação e propor alternativas.

Vale acrescentar que a pandemia recoloca a ciência em evidência na grande mídia e no imaginário coletivo, lhe reatribuindo um valor positivo, combalido pelos ataques negacionistas que marcam os tempos de pós-verdade. No entanto, no ranking do conhecimento científico, mais uma vez as Ciências Biológicas e Genéticas destacavam-se. No campo da Saúde Coletiva, os epidemiologistas ocupam as mídias devido aos conhecimentos específicos na previsão de cenários da disseminação do coronavírus, reconhecendo a necessidade de uma visada de maior complexidade e conclamando análises das CSH para entender a (re)produção social da pandemia e as desigualdades estruturantes que agravam-na.

Por um lado, pode-se atribuir essa configuração valorativa entre áreas da ciência a aspectos pragmáticos associados às respostas necessárias em um momento de emergência pandêmica, em que a decifração do vírus, na sua biologia e genética, a descoberta de medicamentos eficazes e a produção das taxas e curvas epidemiológicas se fazem imprescindíveis para evitar contágio e mortes, ainda que sob o risco de um olhar unicausal do processo saúde-doença. O tempo da emergência canaliza a intenção de conter uma ameaça a coisas valorosas, como a vida 7, mobilizando técnicas de governança que recorrem à inteligência epidêmica e deflagram políticas de quarentenas e vigilância, estabelecem ritmos frenéticos aos trabalhadores de saúde e financiam as pesquisas das vacinas, tudo isso com o intuito de sufocar a epidemia em "tempo real" 8. Por outro lado, etnografias críticas têm alertado para o risco de que o foco no acontecimento deixe de lado o fato de que a epidemia ocorre em um tempo que se prolonga para além da emergência, além de ocultar os conflitos provocados pela necessidade de agir na imediatez 9 . A emergência epidêmica não se confunde com a experiência concreta das pessoas e grupos, com os efeitos sobre o seu ritmo, imaginário e condições de vida para seguirem as prescrições médicas e sanitárias, além dos desdobramentos dos efeitos de verdade inerentes à biopolítica. Esses aspectos ganham recalcitrâncias interpretativas quando discursos científicos são contrapostos às realidades contemporâneas de infodemia e negacionismo, quando há que ter prudência analítica e situar-se politicamente na defesa de ferramentas críticas que possam questionar as verdades, sem, para isso, perder as lições razoáveis 10 .

Aqui se fundamenta, em um contexto político que desqualifica a ciência e demoniza as teorias críticas, nomeadas como "marxismo cultural", o desconforto que emana dos discursos produzidos pelas CSH que desvelam as bases de produção e reprodução das doenças. Assim, ao analisarem a realidade de adoecimento da COVID-19, descortinam a relação da produção desta doença e de tantos outros agravos à saúde que lhes são simultâneos a condições socioeconômicas e ambientais preexistentes, acirradas com as crises sanitárias. As histórias das epidemias e seus estudos socioantropológicos mostram que elas estão relacionadas a mudanças ecológicas profundas, situações geopolíticas, determinando relações entre agências de saúde global 11 e o que Merril Singer 12 (p. 199-200) denomina de "estruturas subjacentes de relações sociais que moldam arquiteturas sociais complexas de desigualdade".

No Brasil, o solo fértil de produção, reprodução ou agravamento da COVID-19 se conforma em meio à obscena desigualdade social provocada pelo neoliberalismo, pelo racismo estrutural, pela masculinidade tóxica vinculada a uma sociedade patriarcal, pelo desmantelamento mais recente das condições e relações de trabalho, gerando precarização e informalidade até a uberização do trabalho e desemprego avassalador, situações que se agravam com a redução generalizada das políticas de pro- 
teção social, associada a uma das mais draconianas políticas econômicas de austeridade. Esse contexto não se origina na pandemia da COVID-19, apenas revela as múltiplas e simultâneas crises vivenciadas pela sociedade brasileira, econômica, política e sanitária, agravadas por ela.

A isso se soma o aprofundamento exponencial da devastação ambiental com forte correlação ao ataque às populações indígenas e camponesas que sofrem maior impacto na pandemia. Nesse sentido, a gestão federal da pandemia acirrou a lógica neoliberal centrada na assunção dos riscos e exposição das populações à morte, promovendo a sobreposição do negacionismo científico e autoritarismo ao discurso neoliberal 13 .

Embora haja uma considerável produção na área das CSH abordando aspectos relacionados à pandemia da COVID-19 14,15,16,17,18,19,20,21,22, permanece flagrante a necessidade de criação de agenda de pesquisas específicas que identifiquem temáticas-chave na compreensão desse problema e aprofundem a investigação dessas realidades na durée. Não há dúvidas de que o estado de pandemia apenas deflagrou, ou agravou, problemas que permanecerão sem solução se não forem adequadamente acessados e enfrentados. É sabido que pesquisas das CSH têm feito avançar o conhecimento sobre as epidemias, não raramente produzindo pontos de inflexão nos modos de seu enfrentamento.

Visando analisar criticamente essa situação e refletir sobre as diversas vertentes pelas quais os PPGs da Saúde Coletiva vislumbrariam contribuições das CSHS para o enfrentamento da pandemia da COVID-19, a CCSHS-Abrasco deflagrou consultas e discussões com os representantes dos mais de 28 programas brasileiros da área. Um dos produtos desse movimento foi a elaboração coletiva de um Termo de Referência (TR) que explicita as nossas matrizes de pensamento. Assim, afirmamos 23 (p. 1):

"Na área das ciências sociais e humanas em saúde, parte-se do pressuposto que a tríade saúde-doençacuidados é mediada por contextos culturais e sociopolíticos, condições materiais e de vida, interações sociais, experiências pessoais, fatores históricos, modelos econômicos e inovação tecnológica. Tendo ainda em vista as características plurais que a pandemia tem assumido nas diversas partes do mundo, tanto nos tempos e formas com que ela tem sido gerida nos seus macro e microcontextos, no nível estatal e da sociedade civil, como também nas populações que ela tem atingido com menor ou maior gravidade, informadas pelas mais recentes tendências epidemiológicas que têm demonstrado que a pandemia cresce entre as populações mais vulneradas nos vários países, é preciso que seja proposta uma agenda de pesquisas que dê conta de analisar essa complexidade. O estudo dessas mediações produz conhecimentos que devem orientar medidas de proteção e formas de prevenção para a pandemia, mas também modos de lidar com o sofrimento que ela produz, além de iluminar novos horizontes de possibilidades para a vida associada às suas múltiplas ondas, e ao que pode se enunciar como um espaço-tempo da pós-pandemia”.

Sintetizamos o TR com a necessidade de explorar e examinar eixos temáticos que já se revelavam campos de produção de conhecimento relevantes na compreensão dos condicionantes e dinâmicas de apresentação da COVID-19, como os que se seguem:

(1) Ciência e COVID-19: práticas e outras epistemologias;

(2) Estado e COVID-19: políticas e o governo da pandemia;

(3) Discriminação e COVID-19: racismo, relações de gênero e desigualdades sociais;

(4) Trabalho e COVID-19;

(5) Violência e COVID-19;

(6) Sofrimento psíquico e COVID-19: produção de subjetividades, formas de sociabilidade e resistência;

(7) Risco e COVID-19: transversalidade com outros eventos de saúde/doença;

(8) Cuidado, bioética e COVID-19; e

(9) Emergências globais e COVID-19.

Analisamos esse processo como uma mobilização que respondeu a necessidades fundamentais de sanitaristas cientistas sociais em saúde em um momento histórico e conjuntura precisos. Primeiramente, havia um mandato de conhecer para elaborar o estado de perplexidade que experimentávamos diante do fenômeno excepcional pela sua raridade e pela violência dos seus efeitos. Em segundo lugar, nos agregávamos à indiscutível urgência de conhecer para agir sobre a realidade pandêmica como analisadores críticos da realidade social que ela gerava e desvelava. Em terceiro lugar, havia uma exigência de denunciar e resistir aos ataques autoritários e antidemocráticos que visavam silenciar, especialmente, vozes críticas de arranjos de desigualdade e opressão. Por fim, definíamos uma atitude afirmativa e propositiva, discutindo concretamente, inclusive nos seus limites, em que se situaria nos- 
sa capacidade de colaborar. Fazíamos, assim, um movimento de re(existência) constitutiva de saberes, práticas e identidade, para dentro, no campo da saúde coletiva e, para fora, no espaço científico.

$\mathrm{O}$ TR foi encaminhado às agências públicas brasileiras de fomento científico (MCTIC, Departamento de Ciência e Tecnologia do Ministério da Saúde - DECIT/MS, e fundações estaduais de amparo à pesquisa - FAPs), para requerer financiamento especificamente para a área e afirmar, politicamente, o lugar das CSHS na análise da pandemia nas suas dimensões sociais, ecológicas, econômicas, culturais e políticas, visando soluções práticas. Desde o início, sabíamos dos limites do alcance dessa estratégia. Até o momento só recebemos resposta do CNPq, informando ter recebido com "satisfação e atenção a proposta da Abrasco", mas que se vê "premido pelas limitações orçamentárias federais para ações de maior vulto”. Não obstante, julgamos que a mobilização de tantos atores sociais e a decisão de ocupar espaços e dar visibilidade à produção e potencialidades dessa área, para fazer face a uma das principais questões humanitárias do século XXI, nos agrega solidariamente e nos fortalece politicamente para seguirmos na disposição de lutar e antecipar projetos para um mundo mais positivamente transformado pelas "lições do vírus" 24 .

\section{Colaboradores}

Todos os autores participaram igualmente da produção do artigo e aprovaram a versão final a ser publicada.

\section{Informações adicionais}

ORCID: Mônica de Oliveira Nunes (0000-00025905-4199); Suely Deslandes (0000-0002-70623604); Marta Inez Machado Verdi (0000-00017090-9541); Rui Massato Harayama (0000-00027231-3589); Luís Henrique da Costa Leão (00000003-0166-5066); Martinho Braga Batista e Silva (0000-0003-3577-958X); Tatiana Engel Gerhardt (0000-0001-8707-6347).

\section{Referências}

1. Petrou C. Guest post - scientific output in the year of COVID. The Scholarly Kitchen 2020; 19 nov. https://scholarlykitchen.sspnet. org/2020/11/19/guest-post-scientific-outputin-the-year-of-covid/.

2. Barbosa L. Brasil ocupa a 11o posição no ranking mundial de estudos sobre Covid. Jornal Opção 2020; 23 nov. https://www.jornalop cao.com.br/ultimas-noticias/brasil-ocupa-a11 o-posicao-no-ranking-mundial-deestudos-sobre-covid-297880/.

3. Coordenação de Aperfeiçoamento de Pessoal de Nível Superior. Portaria no 34, de 9 de março de 2020. Dispõe sobre as condições para fomento a cursos de pós-graduação stricto sensu pela Diretoria de Programas e Bolsas no País da CAPES. Diário Oficial da União 2020; 18 mar.

4. Associação Nacional de Pós-graduação e Pesquisa em Geografia; Associação Nacional de Pós-graduação em Ciências Sociais; Associação Nacional de História; Associação Nacional de Pós-graduação e Pesquisa em Letras e Linguística; Associação Nacional de Pesquisa e Pós-graduação em Psicologia; Associação Brasileira de Antropologia; et al. Relato e reflexões sobre a reunião SBPC/sociedades científicas e governo. O futuro da ciência brasileira? http:// www.aba.abant.org.br/files/20200327_5e7e 3f4205c77.pdf (acessado em 16/Set/2021). 
5. Ministério da Ciência, Tecnologia, Inovações e Comunicações. Portaria no 1.329 , de 27 de março de 2020. Altera a A Portaria no 1.122, de 19 de março de 2020, que define as prioridades, no âmbito do Ministério da Ciência, Tecnologia, Inovações e Comunicações (MCTIC), no que se refere a projetos de pesquisa, de desenvolvimento de tecnologias e inovações, para o período 2020 a 2023. Diário Oficial da União 2020; 30 mar.

6. Associação Brasileira de Saúde Coletiva. Precisamos das Ciências Sociais e Humanas para compreender e enfrentar a pandemia de COVID-19. https://www.abrasco.org.br/site/ wp-content/uploads/2020/04/Precisamosdas-Ci\%C3\%AAncias-Sociais-e-Humanas-pa ra-compreender-e-enfrentar-a-pandemia-deCOVID-19.pdf (acessado em 16/Set/2021).

7. Beckett G. The politics of emergency. Reviews in Anthropology 2013; 42:85-101.

8. Caduff C. Sick weather ahead: on data-mining, crowd-sourcing and white noise. Cambridge Journal of Anthropology 2014; 32:32-46.

9. Roth E. Epidemic temporalities: a concise literature review. Anthropol Today 2020; 36:13-6.

10. Camargo Jr. KR, Coeli CM. A difícil tarefa de informar em meio a uma pandemia. Physis (Rio J.) 2020; 30:e300203.

11. Cueto M. Cold war, deadly fevers. Malaria eradication in Mexico, 1955-1975. Baltimore: Johns Hopkins University Press; 2007.

12. Singer M. Pathogens gone wild? Medical anthropology and the "swine flu" pandemic. Med Anthropol 2009; 28:199-206.

13. Caponi S. Covid-19 no Brasil: entre o negacionismo e a razão neoliberal. Estud Av 2020; 34:209-24

14. Agamben G, Zizek S, Nancy JL, Berardi F, Petit SL, Butler J, et al. Sopa de Wuhan: pensamiento contemporáneo en tiempos de pandemias. ASPO (Aislamiento Social Preventivo y Obligatorio); 2020. https://bit.ly/sopadewuhan.

15. Arévalo A. Presentación. Reflexiones Ístmicas Centroamericanas 2020; (1):5-7.
16. Carrara S. As ciências humanas e sociais entre múltiplas epidemias. Physis (Rio J.) 2020; 30:e300201.

17. Enguita-Fernandez C, Marbán-Castro E, Manders O, Maxwell L, Matta GC. The COVID-19 epidemic through a gender lens: what if a gender approach had been applied to inform public health measures to fight the COVID-19 pandemic? Soc Anthropol 2020; [Epub ahead of print].

18. Grisotti M. Pandemia de Covid-19: agenda de pesquisas em contextos de incertezas e contribuições das ciências sociais. Physis (Rio J.) 2020; 30:e300202.

19. Harvey D. Anti-capitalist politics in the time of COVID-19. Jacobin 2020; 20 mar. https:// jacobinmag.com/2020/03/david-harvey-coro navirus-political-economy-disruptions.

20. Karampampas P. Partying at times of crises and pandemics: solidarity, resilience and coping with the measures against COVID-19. Soc Anthropol 2020; [Epub ahead of print].

21. Martinez-Hernaez A. Um mundo desvalido? In: Evangelidou S, Martínez-Hernáez A, editores. RESET. Reflexiones antropológicas ante la pandemia de COVID-19. Tarragona: URV Publicacions; 2020. p. 27-8

22. Ortega F, Béhague DP. O que a medicina social latino-americana pode contribuir para os debates globais sobre as políticas da Covid-19: lições do Brasil. Physis (Rio J.) 2020; 30:e300205.

23. Comissão de Ciências Sociais e Humanas, Associação Brasileira de Saúde Coletiva. Termo de referência: agenda de pesquisas em ciências sociais e humanas em saúde para a COVID-19. https://www.abrasco.org.br/site/ comissaodecienciassociaisehumanasemsaude/wp-content/uploads/sites/5/2021/02/Ter mo-de-Referencia_-Agenda-de-pesquisas-emciencias-sociais-e-humanas-em-saude-para-aCOVID-19-1.pdf (acessado em 16/09/2021).

24. Santos BS. A cruel pedagogia do vírus. Coimbra: Almedina; 2020.
Recebido em 25/Jun/2021

Versão final reapresentada em 27/Ago/2021

Aprovado em 31/Ago/2021 